\title{
Quasi-classical localized states in the 2D ferrimagnet
}

\author{
Yury Panov ${ }^{1, \star}$ and Alexander Moskvin ${ }^{1}$ \\ ${ }^{1}$ Ural Federal University, 620002, Mira str. 19, Ekaterinburg, Russia
}

\begin{abstract}
We consider highly anisotropic 2D quantum $s=1 / 2$ (pseudo)magnetic system which is equivalent to the frequently used system of charged hard-core bosons on a square lattice. In the continuous quasi-classical approximation, the types of localized excitations are determined by asymptotic analysis and compared with numerical results. Depending on the homogeneous ground state, the excitations are the ferro and antiferro type vortices, the skyrmion-like topological excitations or linear domain walls.
\end{abstract}

\section{Introduction}

We construct a continuous two-sublattice approximation for the 2D system of charged hard-core $(h c)$ bosons, that is equivalent to the highly anisotropic $s=1 / 22 \mathrm{D}$ magnets with a constant magnetization. Quasi-classical continuous description of the 2D magnetic systems reveals their striking features, namely, the collective localized inhomogeneous states with nontrivial topology and finite excitation energy. These include topological solitons $[5,6]$, magnon drops [7], in- and out-of-plane vortex-antivortex pairs [8], and various spiral solutions [9-11]. Basically these solutions have been obtained for the isotropic and anisotropic ferromagnet. In the system of lattice bosons, these states correspond to an inhomogeneous distribution of the charge and the superfluid densities. We consider the asymptotic behavior of localized solutions which converge to homogeneous solutions at infinity and do find their phase diagram. The results are compared with numerical calculations of the ground-state energy in the quasi-classical approximation.

\section{The model}

Hereafter, in the paper we make use of a pseudospin formalism. For instance, the $S=1$ pseudospin formalism $[3,4]$ developed for the copper oxides implies that the on-site Hilbert space described by three effective valence states of the copper ions, $\mathrm{Cu}^{1+, 2+, 3+}$, or, strictly speaking, of the copper-oxygen clusters $\mathrm{CuO}_{4}^{7-, 6-, 5-}$ in the $\mathrm{CuO}_{2}$ plane. These states correspond to the hole occupation numbers $n_{h}=0,1,2$ and can be considered as components of the pseudospin $S=1$ triplet with projections $M_{S}=-1,0,+1$, respectively. The full pseudospin Hamiltonian [4] includes the two-hole transfer and several types of one-hole transfer terms, but here we use a simplified pseudospin Hamiltonian that takes into account only the two-hole transport:

$$
\mathcal{H}=\Delta \sum_{i} S_{i z}^{2}+V \sum_{\langle i j\rangle} S_{i z} S_{j z}-t \sum_{\langle i j\rangle}\left(S_{i+}^{2} S_{j-}^{2}+S_{i-}^{2} S_{j+}^{2}\right) .
$$

A pair of holes plays the role of one boson in the model, however, the condition $\Delta \rightarrow \infty$ eliminates the on-site state with $n_{h}=1$, so in the limit of $\Delta \rightarrow-\infty$, it is equivalent to the Hamiltonian of charged $h c$ bosons in terms of a pseu$\operatorname{dospin} \vec{\sigma}, \sigma_{z}= \pm 1$ :

$$
\mathcal{H}=-t \sum_{\langle i j\rangle}\left(\sigma_{i+} \sigma_{j-}+\sigma_{i-} \sigma_{j+}\right)+V \sum_{\langle i j\rangle} \sigma_{i z} \sigma_{j z} .
$$

Here $\sigma_{\alpha}, \alpha=x, y, z$ are Pauli matrices, $\sigma_{ \pm}=\left(\sigma_{x} \pm\right.$ $\left.i \sigma_{y}\right) / 2$. The $z$-component of the pseudospin describes the local density of bosons, so that antiferromagnetic $z-z$ exchange corresponds to the repulsive density-density interaction, while isotropic ferromagnetic planar exchange corresponds to the kinetic energy of bosons. The constant total number of bosons leads to the constraint on the total $z$ component of the pseudospin. We define the $n$, as the density of the total doped charge counted from the state with a zero total z-component, or parent $\mathrm{Cu}^{2+}$ state. Then $n$ is the sum of $z$-components of the pseudospin: $\sum_{i} \sigma_{i z}=n N$, where $N$ is the total number of sites. If $\rho$ is the density of $h c$ bosons, then $n$ is the deviation from the half-filling: $\rho=(1+n) / 2$.

The energy functional in a quasi-classical approximation

$$
\begin{gathered}
E=\langle\Psi|\mathcal{H}| \Psi\rangle, \quad|\Psi\rangle=\prod|\psi\rangle_{i}, \\
|\psi\rangle=\cos \frac{\theta}{2} e^{-i \frac{\phi}{2}}|+1\rangle+\sin \frac{\theta}{2} e^{i \frac{\phi}{2}}|-1\rangle,
\end{gathered}
$$

where $| \pm 1\rangle$ are the eigenfunctions of the $\sigma_{z}$, takes the form

$$
\begin{aligned}
\varepsilon=- & \sum_{\langle i, j\rangle} \sin \theta_{i} \sin \theta_{j} \cos \left(\phi_{i}-\phi_{j}\right)+ \\
& +\lambda \sum_{\langle i, j\rangle} \cos \theta_{i} \cos \theta_{j}-\xi\left(\sum_{i} \cos \theta_{i}-n N\right) .
\end{aligned}
$$

\footnotetext{
^e-mail: yuri.pano@urfu.ru
} 
Here we define $\varepsilon=2 E / t, \lambda=2 V / t, \xi=2 \mu / t$, where the chemical potential $\mu$ takes into account the bosons density constraint, and $\langle i, j\rangle$ denotes summation over nearest neighbors in a square lattice. The $\theta_{i}$ and $\phi_{i}$ are the polar and azimuthal angles of the quasiclassical pseudospin vector at an $i$-th site.

Hereafter, we introduce two sublattices $A$ and $B$ with the checkerboard ordering. The energy functional (4) in the two-sublattices continuous approximation takes the form

$$
\begin{gathered}
\varepsilon=\frac{1}{2} \int\left[\left(\cos \theta_{A} \cos \theta_{B} \cos \left(\phi_{A}-\phi_{B}\right)-\right.\right. \\
\left.-\lambda \sin \theta_{A} \sin \theta_{B}\right)\left(\nabla \theta_{A}, \nabla \theta_{B}\right)+ \\
+\sin \theta_{A} \sin \theta_{B} \cos \left(\phi_{A}-\phi_{B}\right)\left(\nabla \phi_{A}, \nabla \phi_{B}\right)- \\
-\sin \theta_{A} \cos \theta_{B} \sin \left(\phi_{A}-\phi_{B}\right)\left(\nabla \phi_{A}, \nabla \theta_{B}\right)+ \\
+\cos \theta_{A} \sin \theta_{B} \sin \left(\phi_{A}-\phi_{B}\right)\left(\nabla \theta_{A}, \nabla \phi_{B}\right)+ \\
+4\left(-\sin \theta_{A} \sin \theta_{B} \cos \left(\phi_{A}-\phi_{B}\right)+\lambda \cos \theta_{A} \cos \theta_{B}\right)- \\
\left.-\xi\left(\cos \theta_{A}+\cos \theta_{B}-2 n\right)\right] \mathrm{d} \vec{r},
\end{gathered}
$$

The Euler equations for this functional have rather complicated form. But we note that the first sum in the Exp.(4) has its lowest value if $\cos \left(\phi_{i}-\phi_{j}\right)=1$. This allows us to make a simplifying assumption, that $\phi_{A}(\vec{r})=\phi_{B}(\vec{r}) \equiv \phi(\vec{r})$. It is worth to note that this assumption is confirmed by the results of our numerical simulations. We define functions $u(\vec{r}) \equiv \theta_{A}(\vec{r}), v(\vec{r}) \equiv \theta_{B}(\vec{r})$, and their combinations $f=\cos u \cos v, F=-f+\lambda f_{u v}$, where subscripts $u$ and $v$ denote derivatives with respect to these quantities. Then the Euler equations in the continuous approximation take a compact form

$$
\left\{\begin{array}{l}
f_{u v} \Delta \phi-2 f_{v} \cdot(\nabla u, \nabla \phi)=0 \\
f_{u v} \Delta \phi-2 f_{u} \cdot(\nabla v, \nabla \phi)=0 \\
F \Delta u+F_{u} \cdot(\nabla u)^{2}-f_{u} \cdot(\nabla \phi)^{2}-4 F_{u}+\xi \sin v=0 \\
F \Delta v+F_{v} \cdot(\nabla v)^{2}-f_{v} \cdot(\nabla \phi)^{2}-4 F_{v}+\xi \sin u=0
\end{array}\right.
$$

These equations need to add the bosons density constraint. With the relevant exchange constants, the equations (6) lead to the equations of $[12,13]$.

\section{The asymptotic behavior of localized solutions}

The system (6) along with the boson density constraint has constant (homogeneous) solutions, $\phi=\phi_{0}, u=$ $u_{0}, v=v_{0}$, that determine the well-known ground-state phase diagram [14] of the $h c$ bosons system in the meanfield approximation. Correspondence between phases of anisotropic magnet and $h c$ bosons is given in table 1 .

Given $\lambda<1$ or $n^{2}>(\lambda-1) /(\lambda+1)$ the ground state of the system is a ferromagnetic (FM) (superfluid (SF))

$$
\cos u_{0}=\cos v_{0}=n, \quad \varepsilon_{0}=-2+2(\lambda+1) n^{2} .
$$

\begin{tabular}{ll}
$h c$ bosons & anisotropic magnet \\
\hline superfluid (SF) & ferromagnetic (FM) \\
supersolid (SS) & ferrimagnetic (FIM) \\
charge-ordered (CO) & antiferromagnetic (AFM)
\end{tabular}

Table 1. The corresponding phases of $h c$ bosons and anisotropic magnet.

Given $n^{2}<(\lambda-1) /(\lambda+1)$ the ground state is a ferrimagnetic (FIM) (supersolid (SS)):

$$
\begin{aligned}
\cos u_{0} & =n+z, \quad \cos v_{0}=n-z, \\
\varepsilon_{0} & =-2 \lambda+4|n| \sqrt{\lambda^{2}-1} .
\end{aligned}
$$

where $z^{2}=1+n^{2}-2|n| \lambda / \sqrt{\lambda^{2}-1}$. In all phases, the value of $\xi$ satisfies the regular expression $\xi_{0}=\partial \varepsilon_{0} / \partial n$. When $\lambda>1$ and $n=0$ the FIM phase transforms into a conventional antiferromagnetic (AFM) (charge-ordered (CO)) phase with the checkerboard ordering.

The equations (6) in the case of $\lambda<0$ have localized solutions with nonzero topological charge and finite energy [5-11]. However, in our case $\lambda>0$, numerical calculations with the conjugate gradient method for minimizing of the energy functional (3) on the lattice $256 \times 256$ indicate the existence of similar solutions, at least, as metastable states. The results are shown in figure 1. The actual stability of these solutions in our calculation was different. The FM-phase solutions, similar to that of in figure 1, cases 1 and 2, quickly evolved to an uniform one. The FIM- and AFM-phase solutions, similar to that of in figure 1, cases 3 and 4 , retain its form for more than $10^{6}$ iterations.

We investigated the asymptotic behavior of localized solutions, suggesting that at $r \rightarrow \infty$ they have the form $\phi(\vec{r})=\phi_{0}+\tilde{\phi}(\vec{r}), u(\vec{r})=u_{0}+\tilde{u}(\vec{r}), v(\vec{r})=v_{0}+\tilde{v}(\vec{r})$, where $\tilde{\phi}, \tilde{u}, \tilde{v} \rightarrow 0$ at $r \rightarrow \infty$. Hereinafter, the index 0 means the corresponding values for constant solutions. The linearized system (6) for the functions $\tilde{\phi}, \tilde{u}, \tilde{v}$ takes the form

$$
\left\{\begin{array}{l}
f_{u v 0} \Delta \tilde{\phi}=0, \\
F_{0} \Delta \tilde{u}+4 F_{0} \tilde{u}+4\left(-F_{u v 0}+\frac{\xi_{0}}{4} \cos v_{0}\right) \tilde{v}=0, \\
F_{0} \Delta \tilde{v}+4 F_{0} \tilde{v}+4\left(-F_{u v 0}+\frac{\xi_{0}}{4} \cos u_{0}\right) \tilde{u}=0 .
\end{array}\right.
$$

In the case of the FM and FIM phases, the solutions for the first equation can be written as

$$
\tilde{\phi}(\vec{r})=\sum_{m=1}^{\infty} \frac{c_{m}}{r^{m}} \cos m\left(\varphi-\varphi_{m}\right),
$$

with $c_{m}$ and $\varphi_{m}$ determined by the boundary conditions. In the case of the AFM phase, the first equation reduces to an identity since $f_{u v 0}=0$.

In the case of the FM phase, the second and the third equations become independent Helmholtz equations for the ferro- and antiferro-type combinations $U=\tilde{u}+\tilde{v}$, $V=\tilde{u}-\tilde{v}$ :

$$
\Delta U+A_{1} U=0, \quad A_{1}=4 \frac{(\lambda+1)\left(1-n^{2}\right)}{\lambda-(\lambda+1) n^{2}} ;
$$




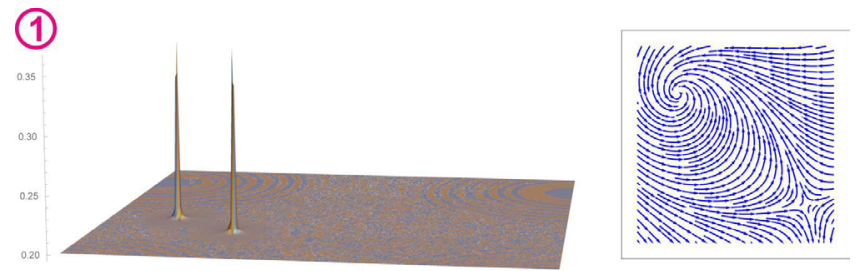

(2)
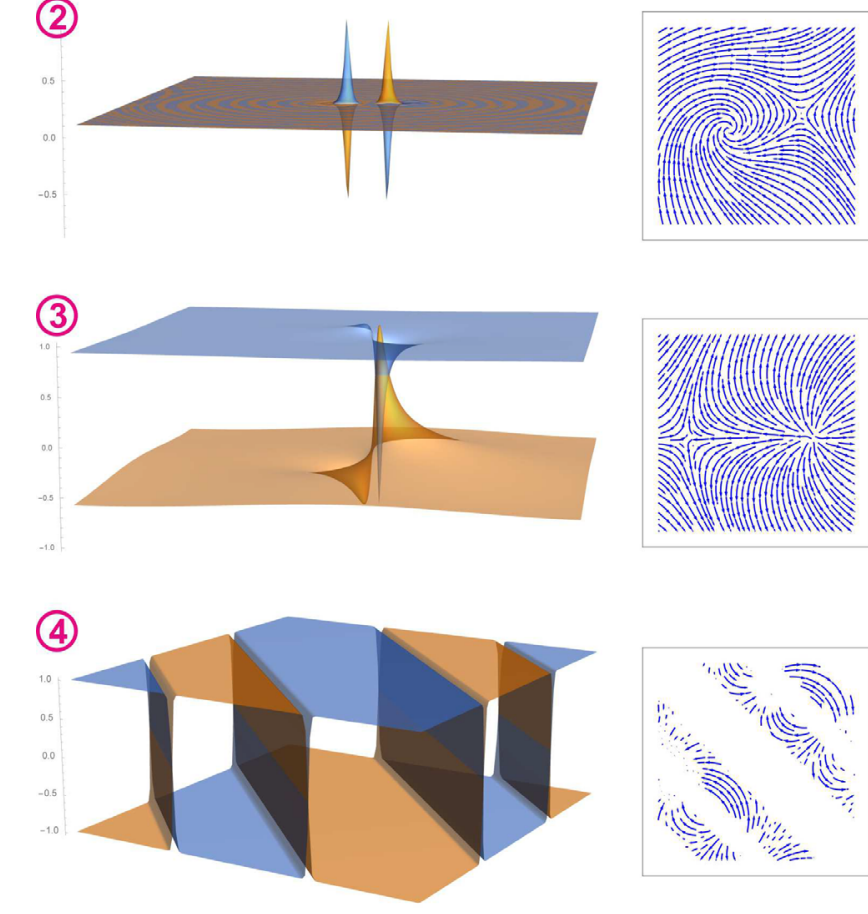

Figure 1. (color online) Inhomogeneous states in 2D system of charged hard-core bosons. The left panels show local charge density $n_{i}=\sigma_{z i}=\cos \theta_{i}$. The difference of sublattice states is clearly evident in the cases 2, 3 and 4 . The right panels show phase flow of planar components of the pseudospin, $\sigma_{x i}$ and $\sigma_{y i}$. The phase flow reveals the vortex-antivortex pair structure in the core of inhomogeneity of the local charge density in the cases 1,2 and 3 . The parameters of the model are: 1) $n=0.2, \lambda=0.5$ (FM phase); 2) $n=0.1, \lambda=0.9$ (FM phase); 3 ) $n=0.2, \lambda=1.5$ (FIM phase); 4) $n=0.0, \lambda=1.5$ (AFM phase). These sets of parameters are denoted with numbers 1-4 on the ground-state phase diagram in figure 2 .

$$
\Delta V+A_{2} V=0, \quad A_{2}=4 \frac{\lambda-1-(\lambda+1) n^{2}}{\lambda-(\lambda+1) n^{2}} .
$$

The solutions has the form

$$
\begin{aligned}
\Phi\left(\vec{r}, r_{i}\right)= & \sum_{l=0}^{\infty} a_{l} K_{l}\left(r / r_{i}\right) \cos l\left(\varphi-\alpha_{l}\right), \\
\Psi\left(\vec{r}, r_{i}\right)= & \sum_{l=0}^{\infty}\left[b_{1 l} J_{l}\left(r / r_{i}\right) \cos l\left(\varphi-\beta_{1 l}\right)+\right. \\
& \left.+b_{2 l} Y_{l}\left(r / r_{i}\right) \cos l\left(\varphi-\beta_{2 l}\right)\right],
\end{aligned}
$$

where $K_{l}$ are the Macdonald functions, $J_{l}$ and $Y_{l}$ are the Bessel functions of the first and second kind, and $a_{l}, \alpha_{l}$, $b_{k l}, \beta_{k l}, k=1,2$ are some constants. An analysis of the asymptotic behavior of solutions $(13,14)$ and the requirement that the omitted nonlinear terms in equations (9) are small in comparison with the remaining linear terms determine that $\Psi=0$. The account in the lowest order of the mixing with the function $\tilde{\phi}$ does not change $V(\vec{r})$ and gives additional term in $U(\vec{r})$ having asymptotic behavior:

$$
U_{1}(\vec{r}) \approx-\frac{n c_{m}^{2}}{2(\lambda+1) \sqrt{1-n^{2}}} \frac{m^{2}}{r^{2 m+2}},
$$

where $m$ is the number that specifies first nonzero term in (10).

Line $n^{2}=\lambda /(\lambda+1)$ is the boundary of areas of the FMphase with a different behavior of the $U$ and $V$ functions

$$
\begin{aligned}
& n^{2}>\frac{\lambda}{\lambda+1}: U(\vec{r})=\Phi\left(\vec{r}, r_{1}\right)+U_{1}(\vec{r}), \quad V(\vec{r})=0 ; \\
& n^{2}<\frac{\lambda}{\lambda+1}: U(\vec{r})=U_{1}(\vec{r}), \quad V(\vec{r})=\Phi\left(\vec{r}, r_{2}\right) .
\end{aligned}
$$

Here we define characteristic lengths $r_{i}^{-2}=\left|A_{i}\right|$.

In the case of the FIM phase, we need to define the ferri-type combinations: $\tilde{U}=A \tilde{u}+\tilde{v}$ and $\tilde{V}=A \tilde{u}-\tilde{v}$, where $A=-F_{u v 0}+\frac{\xi_{0}}{4} \cos u_{0}$. The equations (9) lead to Helmholtz equation for the $\tilde{U}$ function having solution $\Psi\left(\vec{r}, r_{3}\right), r_{3}^{-2}=$ 8. As in previous case we have to put $\Psi=0$. The $\tilde{V}$ function obeys to Laplace equation. Taking into account the mixing in the lowest order with the function $\tilde{\phi}$ we come to the expressions

$$
\begin{gathered}
\tilde{U}(\vec{r})=\frac{c_{m}^{2}}{8}\left(A f_{u 0}+f_{v 0}\right) \frac{m^{2}}{r^{2 m+2}}, \\
\tilde{V}(\vec{r})=\sum_{l=1}^{\infty} \frac{C_{l}}{r^{l}} \cos l\left(\varphi-\gamma_{l}\right)+\frac{c_{m}^{2}}{4}\left(A f_{u 0}-f_{v 0}\right) \frac{1}{r^{2 m}},
\end{gathered}
$$

where $m$ is the number that specifies first non-zero term in (10), and the expressions $f_{u 0}=-\sin u_{0} \cos v_{0}, f_{v 0}=$ - $\cos u_{0} \sin v_{0}$ are determined by the expressions (8).

Similarly the case of the AFM phase, we obtain

$$
\tilde{\phi}(\vec{r})=0, \quad U(\vec{r})=0, \quad V(\vec{r})=\Phi\left(\vec{r}, r_{4}\right),
$$

where $r_{4}^{-2}=4(\lambda-1)$.

\section{Discussion}

The analysis of the asymptotic behavior of the localized states reveals qualitative differences of the finite energy excitations in the FM, FIM, and AFM phases.

In the FM phase an asymptotic of the polar angle of the pseudospin vector is determined by the expressions (16, 17). When comparing these results with numerical calculations it is worth to note that the characteristic lengths obey to inequality $r_{i}<1$ in the most part of the phase diagram in the SF phase except for the areas indicated shadowed in figure 2, so the function $\Phi$ goes to zero value very fast with increasing of $r$. On the contrary, the asymptotic behavior of the azimuthal angle of the pseudospin (10) has no characteristic scale. This means that in the FM phase the main excitations are almost in-plane vortex-antivortex pairs. They have well localized out-of-plane core of the ferro type, with $\sigma_{z A}=\sigma_{z B}$, as shown in figure 1, and become the pure in-plane ones at $n=0$ in accordance with 


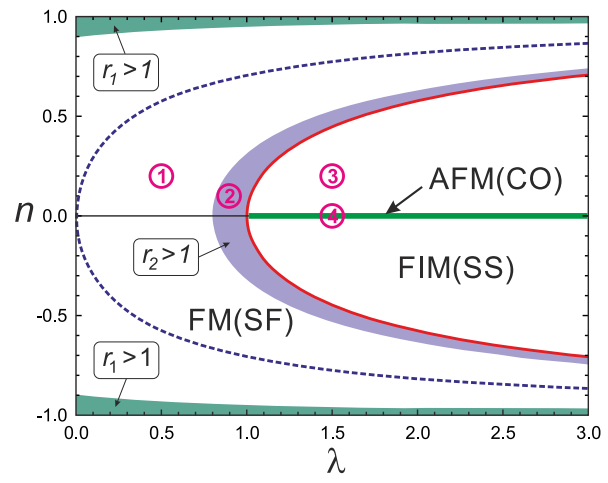

Figure 2. (color online) The ground state phase diagram of the $h c$ bosons in the mean-field approximation. The solid line corresponds to SF-SS phase boundary, $n^{2}=(\lambda-1) /(\lambda+1)$. The thick line at $\lambda>1, n=0$ shows CO phase. The dotted line, $n^{2}=\lambda /(\lambda+1)$, separates the two types of the asymptotic behavior in accordance with the expressions (16) and (17). In the shaded areas inside the SF phase region the characteristic lengths satisfy to inequalities $r_{i}>1$. The numbers 1-4 in the circles correspond to the parameters sets in figure 1 .

expression (15). The same type of localized solutions was found by the authors of [8]. For the $h c$ bosons, the polar angle is related with the density of bosons, while the azimuthal angle is responsible for the superfluid density, hence these states correspond to the excitation of the superfluid component with highly localized heterogeneity of bosons density in the foci of the vortex-antivortex pairs. In the shaded region in the FM phase near the border of the FM-FIM phases in figure 2, the antiferro type vortices, with $\sigma_{z A} \neq \sigma_{z B}$, begin to dominate, their inflation is preceded by a change of the homogeneous ground state from FM to FIM phase.

The AFM phase has no linear excitation of $\tilde{\phi}$. The characteristic lengths of the azimuthal excitations (20) are small except the region near $\lambda=1$. This results in a high stability of the homogeneous AFM phase. A typical picture of the inhomogeneous state shown in figure 1 is represented by linear domains of the AFM phase. The non-zero values of the $\mathrm{FM}(\mathrm{SF})$ order parameter are realized within the domain walls, thus giving rise to appearance of a filamentary superfluidity in the $h c$ bosons.

In the FIM phase the asymptotic behavior of the polar and azimuthal excitations is qualitatively the same with- out characteristic scales. Hence in this case there are skyrmion-like excitations as shown in figure 1. For the $h c$ bosons these coherent states include both the excitations of the superfluid component and the boson density. In a center of skyrmion the difference $\sigma_{z A}-\sigma_{z B}$ has maximal magnitude, that corresponds to $\operatorname{AFM}(\mathrm{CO})$ phase, and near there is a region where $\sigma_{z A}-\sigma_{z B}=0$, that corresponds to FM(SF) phase. So, the skyrmion-like excitations in the FIM(SS) phase generate the topological phase separation. Note, that another type of instability in FIM(SS) phase were also found by Quantum Monte Carlo calculations [15].

The work supported by Act 211 Government of the Russian Federation, agreement No 02.A03.21.0006 and by the Ministry of Education and Science, projects 2277 and 5719.

\section{References}

[1] T. Matsubara, H. Matsuda, Progr. Theor. Phys. 16, 569 (1956)

[2] E. Kim, M.H.W. Chan, Nature 427, 225 (2004)

[3] A.S. Moskvin, Phys. Rev. B 84, 075116 (2011)

[4] A.S. Moskvin, JETP 121, 477 (2015)

[5] A.A. Belavin, A.M. Polyakov, JETP Letters, 22, 245 (1975)

[6] V.P. Voronov, B.A. Ivanov, A. Kosevich, Sov. Phys.: JETP 57, 1303 (1983)

[7] B.A. Ivanov, A.M. Kosevich, Sov. Phys.: JETP 45, 1050 (1977)

[8] M.E. Gouvea, G.M. Wysin, A.R. Bishop, F.G. Mertens, Phys. Rev. B 39, 11840 (1989)

[9] A.B. Borisov, JETP Letters 73, 242 (2001)

[10] I.G. Bostrem, A.S. Ovchinnikov, JETP Letters 76, 716 (2002)

[11] A.B. Borisov, I.G. Bostrem, A.S. Ovchinnikov, JETP Letters 80, 103 (2004)

[12] I. Bostrem, A. Ovchinnikov, R. Egorov, Physics Letters A 279, 33 (2001)

[13] R. Egorov, I. Bostrem, A. Ovchinnikov, Physics Letters A 292, 325 (2002)

[14] R. Micnas, J. Ranninger, S. Robaszkiewicz, Rev. Mod. Phys. 62, 113 (1990)

[15] G.G. Batrouni, R.T. Scalettar, Phys. Rev. Letters 84, 1599 (2000) 